\title{
S-E-7. Reconsideration of Therapeutic Hypothermia for Severe Head Injury
}

\author{
Tetsuro Miwa, Koreyoshi MaKino, Michitaka Kono, \\ Iwao IWAKI, Masamichi Hasue \\ and Morio SAITo \\ Department of Surgery, Tokyo Medical College
}

The therapeutic hypothermia for severe head injury is a method to save cases in which relief is not possible by other means. This method has been recently introduced actively.

However, the effectiveness as well as the disadvantages of this procedure has been pointed out from the various points.

We have stated as a results of our experimental study, that this procedure is safe for the standpoints of metabolism and circulation, if the rectal temperature is maintained at $30^{\circ} \mathrm{C}$ for approximately 48 hours. We applied this treatment to 19 . out of 40 cases suffering severe head injury over the past 2 years. However, since the results showed a death-rate of 10 cases, we have reconsidered this method, in comparison with the survival-rate in regards to the following points.

1. Stabilizing or suppressive action for unbalanced brain metabolism and circulation.

When these patients were put into a hypothermic condition during definite time-periods, the condition of the patients improved in regards to metabolism and circulation. When this procedure was in excess of the need, the side effects were increased rather than improving the patients condition.

\section{Suppressive action for abnormal fever.}

With the hypothermic procedure, the fever of survival cases showed a stability and the same tendency in type. Of the death-cases, however, this showed an unstability, such as rapid rising of fever or a continuance of a low temperature.

\section{Effect for brain edema.}

When we examined distribution of water contents of brain by ${ }^{24} \mathrm{Na}$ in the experimental brain-edema, we found hypothermia is more effctive than hyperthermia for edema.

However, when treatment is prolonged, it has a tendency to cause further brain edema due to increasing permeability of cell membrane of

$$
-107-
$$


brain. Generally, the hypothermic effects were recognized in far as brain lesion consisted of edema mainly, but when the brain damage was very severe, we didn't find any hypothermic effects.

\title{
4. Pathological changes of other organs except for brain.
}

On the autopsy of clinical and experimental cases, the histological findings showed expression of circulatory disturbances, such as bleeding, hyperemia and stasis, in various organs. And, macroscopical findings showed phenomena of accumulation of fluid in the organs, such as lung edema or juice collection in the stomach cavity. These findings suggest we have to reconsider the maintenance time of hypothermia.

\section{S-E-8. Clinical Studies on the Therapies of Acute Head Injury reducing the Frequency of Posttraumatic Sequelae}

\author{
Haruyuki Kanaya and Kosaku Yoneyama \\ 2nd Dept. of Surgery, Iwate Medical College
}

For the past 8 years, 1491 cases of head injury were treated in our clinic and the effects of posttraumatic sequelae in acute stadium were investigated in 608 follow-ups.

Treatments for acute head injury were performed dividing the cases into the active therapy group including 98 cases, in which treatments for improving hypertension included brain edema, circulatory disturbance and $\mathrm{O}_{2}$ metabolic disturbance caused by head injury were tried, and the conservative therapy group including 361 cases, in which some of those treatments were selectively applied according to the patient's symptoms, and then the results were compared.

1) Of 459 cases which we have treated in acute stadium the frequency of posttraumatic sequelae was $51.4 \%$, and those symptoms were headache $54 \%$, poor memory $43.5 \%$, personality disorder $38.4 \%$, nuchal pain, $35.4 \%$, dizziness, $34.3 \%$, asthenopy, $34.1 \%$, etc.

2) In the active therapy group of the cases of severe head injury, posttraumatic sequelae were less frequent by $5 \%$ than the conservative group and it was $10 \%$ of the cases of slight head injury.

3) The frequency of posttraumatic sequelae of the group with neurological deficits was two times of the group without neurological deficits. The effects of the therapy were almost equal in both active and conservative 